\title{
Two-layer reinforced concrete panels with uniformly compressed sections
}

\author{
Dmitry Mailyan ${ }^{l}$, Alik Blyagoz ${ }^{2, *}$, and Konstantin Kretinin ${ }^{2}$ \\ ${ }^{1}$ Don State Technical University, 162 Sotsialisticheskayastr, Rostov-on-Don, 344022, Russia \\ ${ }^{2}$ Federal State Budgetary Educational Institution of Higher Education «Kuban State Agrarian University named after I.T. Trubilin» \\ 13, Kalininastr, Krasnodar, 350044, Russia
}

\begin{abstract}
The paper contains the issues related to the creation of a non-uniform prestress cross-section of reinforced concrete elements compressed during operation of working with a one-way eccentricity of the resultant longitudinal forces.
\end{abstract}

\section{Introduction}

Compressed reinforced concrete elements, as a rule, have a symmetrically arranged longitudinal reinforcement, so when it is pre-stressed, the symmetrical sections are uniformly compressed. This is reasonable because in most cases, compressed elements are subjected to alternating forces. Nevertheless, a number of compressed reinforced concrete elements in use operate with a one-way eccentricity of the resultant longitudinal forces. These include, for example, the racks of transport galleries, elevated roads, bridges, overpasses, etc. Moreover, the static scheme of such elements can be done so that the deflection moments along the entire length have the same non-changing sign. In these cases, it is advisable to create a non-uniform prestress section of the elements. Part of the external load will be used to offset the initial deflection created in this way, and the total axial eccentricity of the external longitudinal force $N$ will be reduced by the value of the initial deflection $f_{p}$ (Fig. 1), the boundaries of the effective use of prestressing in compressed elements are expanded. As a result of these factors, the load-bearing capacity of compressed elements increases, which makes it possible to obtain significant savings in valve consumption while maintaining its value unchanged $[4,5]$.

\section{Experimental program and research results}

Non-uniform prestress section and the initial deflection of the element in cross-section and reinforcement are symmetric with respect to axis passing through center of gravity perpendicular to the force action plane, and these are formed by inequality of effort prestress in $A_{s p}$ and $A_{s p}^{\prime}$, i.e. at $\sigma_{s p} A_{s p} \neq \sigma_{s p}^{\prime} A_{s p}^{\prime}$. In case of an unsymmetrical arrangement of the rebars the deflection was formed even by the same value of forces in the rebars $A_{\text {sp }}$ and $A_{\text {sp }}^{\prime}$.

Compressed elements include wall panels along with columns, piles, and other structural elements. A progressive solution is the prestressed double-layer wall panels developed by the originators that combine load-bearing and enclosing functions. The use of such panels, whose height is equal to the height of a single-storey industrial- or agricultural building, allows you to abandon the frame solution, which increases prospects of a fully-prefabricated structure by elimination columns, half-timbered pillars, foundation beams, and other elements [8].

The connection between the layers of panels is provided by the adhesion between heavy and light concrete, which, as studies have shown, is quite sufficient and reliable. Concreting of panels is performed in a horizontal position - first, the first layer of heavy concrete is laid, and immediately after that, the second layer of light concrete is laid. After each layer is vibrated, there is some interpenetration of the concrete layers, which helps to increase the adhesion between them.

Pre-compression of the proposed panels, including non-uniform, allows to increase their rigidity and stability, which is especially important at a high height of buildings. The geometric flexibility $\left(l_{0} / \mathrm{h}\right)$ of the panels can reach 30 or more. In addition, pre-compression increases the crack resistance and deformability of panels when operating loads with large eccentricities are applied, as well as when deflection moments are applied during transportation and installation of panels $[6,7]$.

*E-mail: alfa-maikop@,yandex.ru 
With non-uniform pre-compression of panel sections, the initial deflection is formed to one side of the face with less compression (Fig. 1, b).

a)

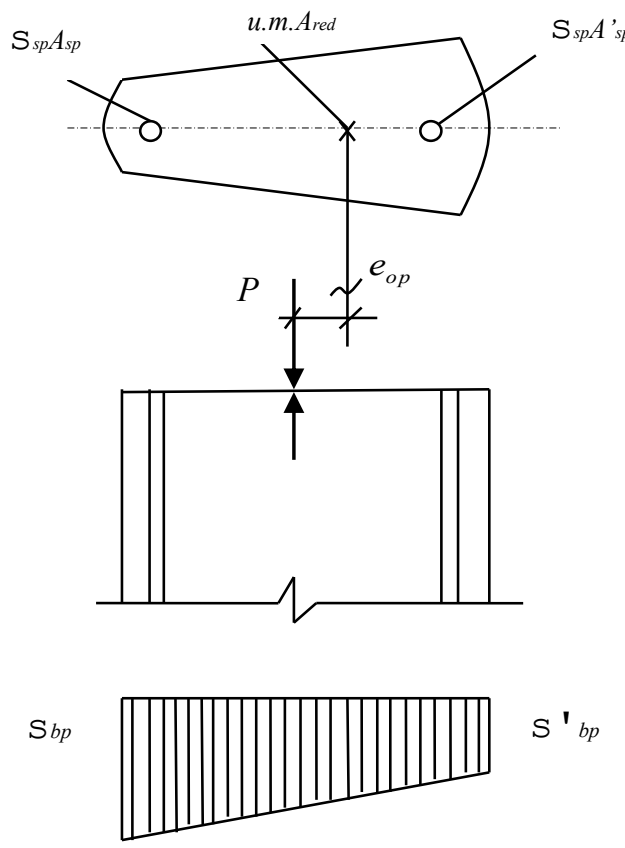

b)

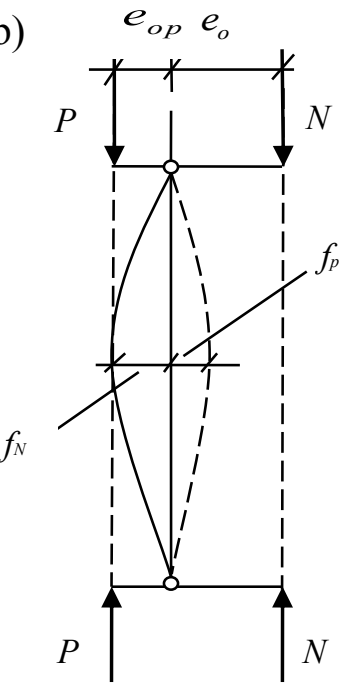

Fig.1. Compressed elements with non-uniformly prestressed sections: a - reinforcement of the section; b - diagram of the operating forces during pre-compression of the section of the element.

Misalignment of the force $N$ from the axis passing through the center of gravity of the section decreases and becomes equal to $\left(\mathrm{e}_{0}+f_{N}-f_{p}\right)$, which leads to a significant reduction in the deflection of the bent panels.

However, this positive effect of non-uniform prestressing of cross sections occurs when the following condition is met:

$$
e_{0}>k f_{p}
$$

where $e_{0}$ - is the axial misalignment of the longitudinal force measured from the axis passing through the center of gravity of the section close to the supports; $f_{p}$ - is the deflection in the middle of the element caused by non-uniform compression; $k$ - is a coefficient depending on the longitudinal force.

To determine a coefficient $k$, included in this condition, we make a differential equation of the elastic axis of the element (Fig. 2):

$$
B\left(y^{\prime \prime}-1 / r_{p}\right)=N\left(\Delta-y-e_{0}\right)
$$

in which the left part is the product of stiffness of the cross sections $B$ by the difference of curvatures from the external force $N$ and the bend, and the right part is the moment from the force $N$ in any cross section of the element in the deformed state.

We represent the solution of equation (2):

$$
y=\frac{1}{\cos \frac{m l}{2}}\left(\frac{\xi}{m^{2}}-e_{0}\right)(1-\cos m x),
$$

where:

$$
m^{2}=N / B \text { and } \xi=1 / r_{p}=8 f_{p} / l^{2}
$$

Let us determine the misalignment $e_{0}$, at which the movement of the end of the rack does not exceed the deflection $f_{p}[4,5]$. To do this, in (3) we substitute the condition $y<f_{p}$ and having in mind (4) after the transformations we get: 


$$
e_{0}>f_{p}\left(\frac{8 B}{N l^{2}}-\frac{\cos \frac{l}{2} \sqrt{\frac{N}{B}}}{1-\cos \frac{l}{2} \sqrt{\frac{N}{B}}}\right)
$$

The coefficient $k$, equal to the expression in parentheses is a function of a free length of the column $l$, cross section stiffness $B$ and longitudinal force $N$.
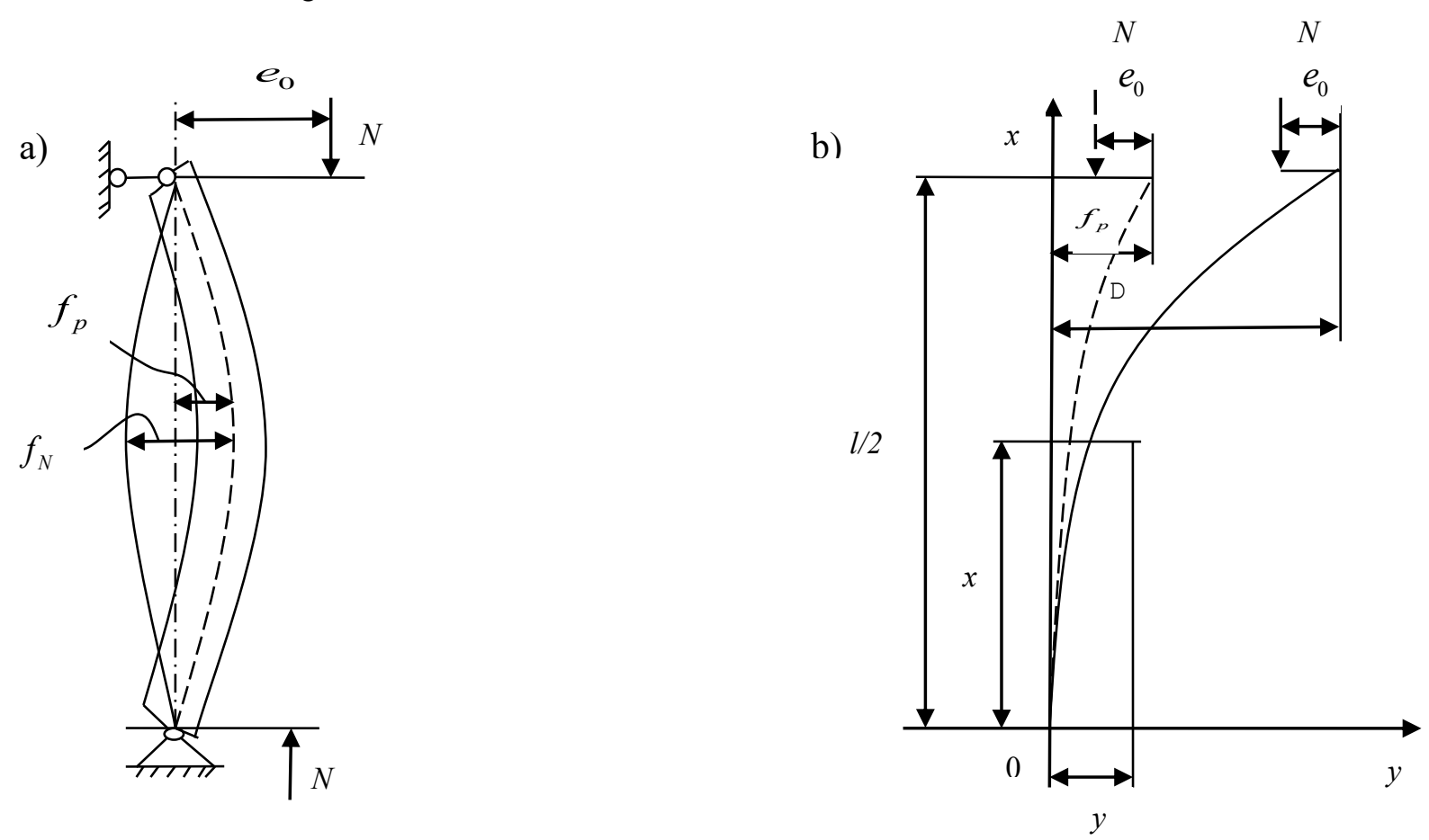

Fig.2.To determine the conditions for the feasibility of creating a non-uniform prestressing of concrete in flexible compressed elements; $a$ - diagram of the panel with hinge supports; $b$ - designations for eccentricity from the elastic axis.

Experiments conducted in the Russian State Social University [1,2] have shown that changes in these parameters over a wide range have little effect on the numerical values of the coefficients: with increase in $N$ and $l / B$ by 3-4 times $k$ decreases from 0.85 up to 0.81 (Fig. 3).

a)

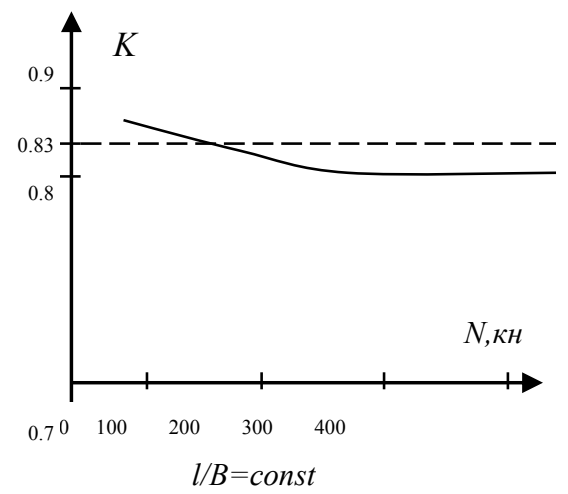

b)

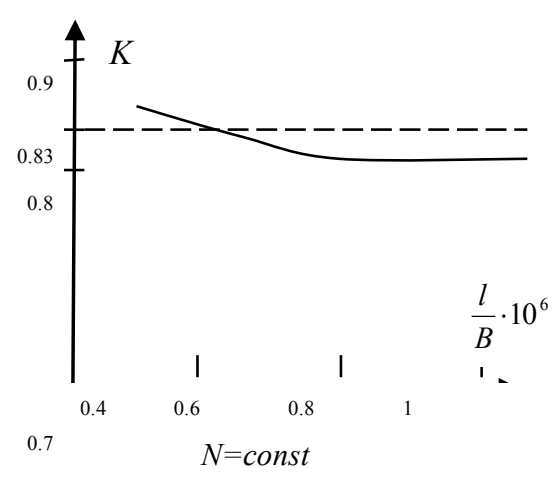

Fig.3. Dependence of coefficient $k$ accordingly on $N\left(\right.$ a) and $l_{0} / B$ (b) respectively to the data [1, 2].

If condition (5) is met, the non-uniform compression significantly increases the relative crack resistance and reduces the deflection of the compressed elements, and given that, the positive effect with an increase in the relative misalignment up to 0.8 or more is practically not reduced.

The results of experiments [1-3] with uniform $\left(\eta=\eta^{\prime}=0.34\right)$ and non-uniform $\left(\eta=0.37 ; \eta^{\prime}=0.15\right)$ compression are shown in Fig. 4, where it can be seen that the non-uniform compression of sections at $e_{0}>0.83 f_{p}$ significantly increases the load-bearing capacity of the compressed elements in comparison with the uniform compression intensity equal to the maximum at the non-uniform. At $e_{0} / h=0.25\left(\mathrm{e}_{0}=2.7 f_{p}\right)$ the bearing capacity of non-uniformly compressed elements is greater than that of uniformly compressed elements by $35 \%$. 
a) $\mathrm{N}_{\mathrm{u}}{ }^{\exp }$

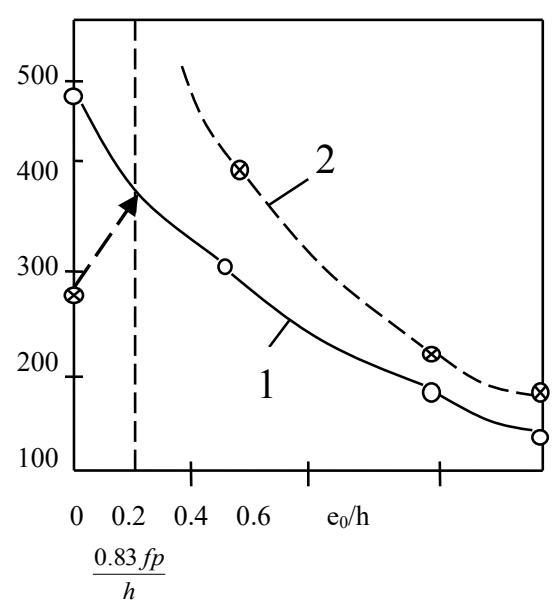

b)
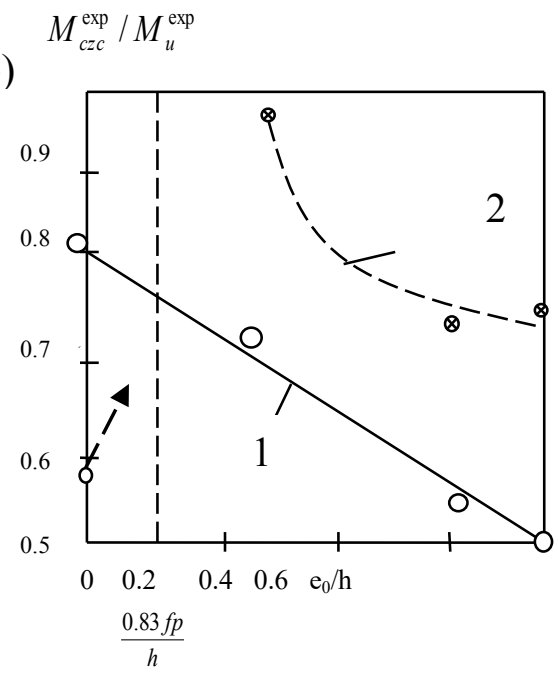

c)

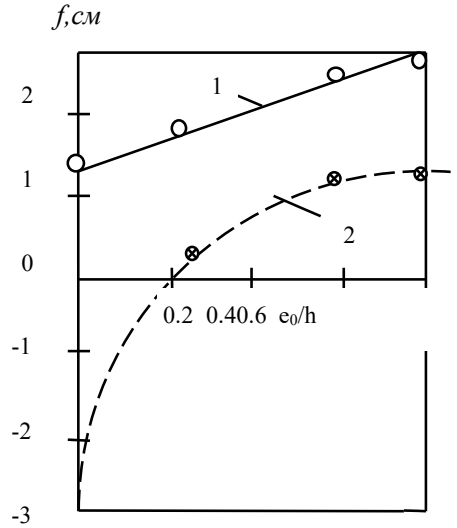

Fig.4. Variation in the load-bearing capacity (a), crawn s esistance (b) and deflections in the midsection at $\mathrm{M}=0.7 \mathrm{Mu}(\mathrm{c})$ with respect to the relative misalignment for the compressed elements with uniformly (1) and non-uniformly (2) pre-compressed sections according to data $[1,2]$.

\section{Conclusion}

With an increase in the misalignment of the external longitudinal force, the load-bearing capacity of non-uniformly compressed elements approaches the load-bearing capacity of uniformly compressed elements and at $e_{0} / h=0.8$ becomes almost equal to the latter. The value of the boundary relative misalignment $e_{0}^{r p} / h$, at which the load capacity of uniformly and non-uniformly compressed elements becomes the same, depends on the level of compression of sections $\eta$ and its difference $\eta / \eta^{\prime}$. As they increase, the boundary value of the relative misalignment increases.

\section{References}

1. D. R. Mailyan Concrete and reinforced concrete 2 (1982)

2. D. R. Mailyan, N. Z. Sukhail Questions of strength, deformability and crack resistance of reinforced concrete (Russian Institute of Strategic Research, Rostov-on-Don, 1986)

3. V. F. Mayboroda, V. M. Karnyuk Three-layer reinforced concrete structures (Budivelnik,Kiev,1990)

4. A. Ryabukhin, A. S. Matsiy In-situ measurement of the anchor-pile displacement in the geotechnical conditions of Sochi Proceedings of the 5th International Young Geotechnical Engineers' Conference (IYGEC 2013) 576-578 (France. Paris: Ecole des PontsParisTech, 2013)

5. A.K. Ryabukhin, V.A. Lesnoy, D.Y. Kalashaov IOP Conf. Ser.: Mat. Sci. and Eng.. 698 (7), 077003 (2019)

6. E. Bezuglova, S. Matsiy Engineering and geological grounds of landslide protection reliability of structures Landslide Science and Practice: Risk Assessment, Management and Mitigation 6, 709-714 (2013)

7. E. Bezuglova, S. Matsiy, V. Podtelkov Landslide risk management at transport facilities Landslides and Engineered Slopes. Experience, Theory and Practice 2, $405-409$ (2016) 
8. S.I. Matsiy, L.A. Sukhlyaeva Geotechnics Fundamentals and Applications in Construction: New Materials, Structures, Technologies and Calculations (Proceedings of the International Conference on Geotechnics Fundamentals and Applications in Construction: New Materials, Structures, Technologies and Calculations, GFAC 2019) 178-181 (2019) 